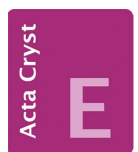

CRYSTALLOGRAPHIC COMMUNICATIONS

ISSN 2056-9890

Received 17 November 2016

Accepted 19 November 2016

Edited by J. Simpson, University of Otago, New Zealand

Keywords: crystal structure; fluroxypyr; herbicide; pyridine; hydrogen bonds.

CCDC reference: 1518035

Supporting information: this article has supporting information at journals.iucr.org/e

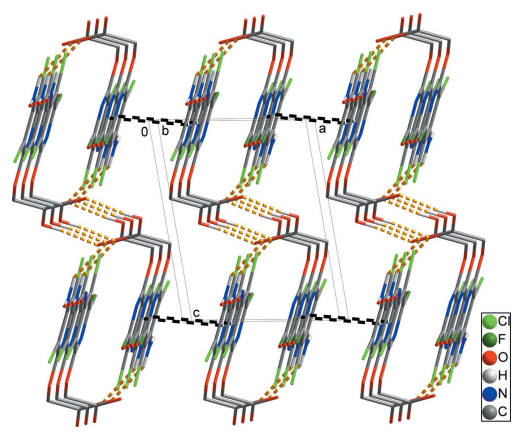

OPEN $\odot$ ACCESS

\section{Crystal structure of fluroxypyr}

\author{
Hyunjin Park, Myong Yong Choi,* Eunjin Kwon and Tae Ho Kim*
}

Department of Chemistry (BK21 plus) and Research Institute of Natural Sciences, Gyeongsang National University, Jinju 52828, Republic of Korea. *Correspondence e-mail: mychoi@gnu.ac.kr, thkim@gnu.ac.kr

In the title pyridine herbicide \{systematic name: 2-[(4-amino-3,5-dichloro-6fluoropyridin-2-yl)oxy]acetic acid\}, $\mathrm{C}_{7} \mathrm{H}_{5} \mathrm{Cl}_{2} \mathrm{FN}_{2} \mathrm{O}_{3}$, the mean plane of the carboxylic acid substituent and the pyridyl ring plane subtend a dihedral angle of $77.5(1)^{\circ}$. In the crystal, pairs of $\mathrm{O}-\mathrm{H} \cdots \mathrm{O}$ hydrogen bonds form inversion dimers with $R_{2}^{2}(8)$ ring motifs. These are extended into chains along [011] by $\mathrm{N}-$ $\mathrm{H} \cdots \mathrm{F}$ hydrogen bonds. In addition, intermolecular $\mathrm{N}-\mathrm{H} \cdots \mathrm{O}$ hydrogen bonds and weak $\pi-\pi$ interactions [ring centroid separation $=3.4602(9) \AA$ ] connect these chains into a three-dimensional network.

\section{Chemical context}

Fluroxypyr belongs to the pyridine family of herbicides. It is widely used on cereal crops, olive trees and fallow croplands to control broad-leaf weeds (Moreno-Castilla et al., 2012; Wang et al., 2011). Pyridine herbicides such as fluroxypyr are effective and popular chemicals for post-emergence broad-leaf weed control, particularly in turf during cool seasons. The efficacy of this herbicide may be affected by environmental conditions including the relative humidity, temperature and soil moisture. Because of this, its application often provides inconsistent broad-leaf weed control in winter or early spring (Reed \& McCullough, 2012). Until now, its crystal structure had not been reported and we describe it herein.

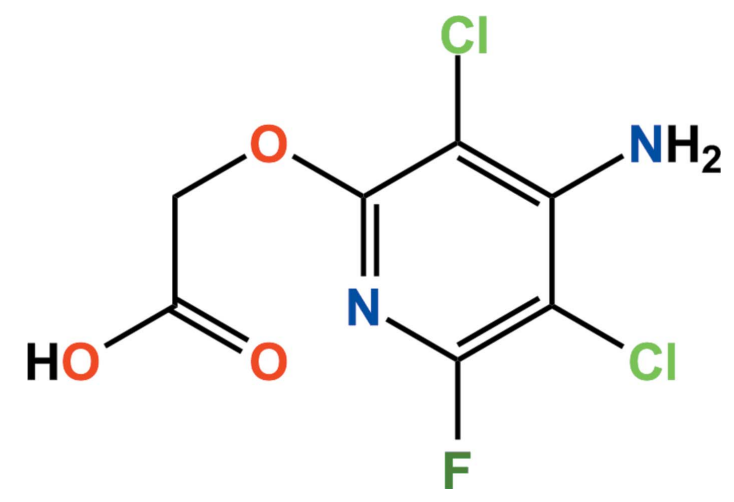

\section{Structural commentary}

The structure of fluroxypyr is shown in Fig. 1. The dihedral angle between the mean plane of the carboxylic acid group $(\mathrm{C} 6 / \mathrm{C} 7 / \mathrm{O} 2 / \mathrm{O} 3)$ and the pyridyl ring $(\mathrm{N} 1 / \mathrm{C} 1-\mathrm{C} 5)$ is $77.5(1)^{\circ}$. All bond lengths and bond angles are normal and comparable to those observed in the crystal structure of a related pyridinecontaining herbicide (Cho et al., 2015). 


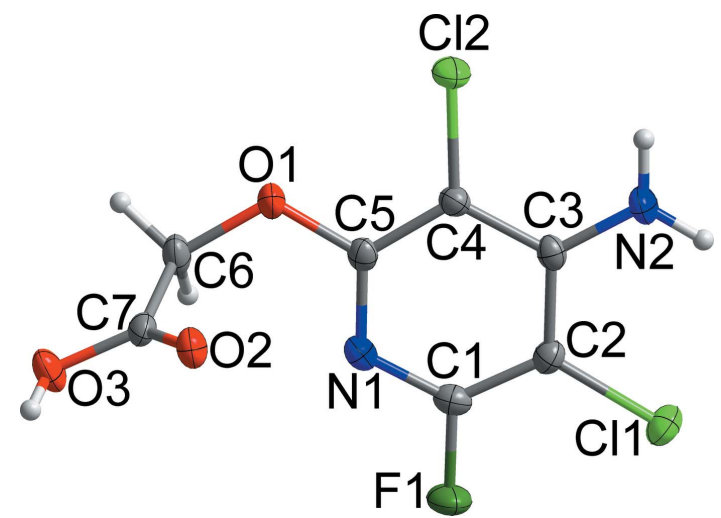

Figure 1

The structure of the title compound, with displacement ellipsoids drawn at the $50 \%$ probability level. $\mathrm{H}$ atoms are shown as small spheres of arbitrary radius.

\section{Supramolecular features}

In the crystal, the solid-state structure is stabilized by pairs of $\mathrm{N} 2-\mathrm{H} 2 B \cdots \mathrm{O} 2$ hydrogen bonds, forming inversion dimers with $R_{2}^{2}(18)$ ring motifs (Table 1 and Fig. 2). These dimers are linked by pairs of $\mathrm{O} 3-\mathrm{H} 3 \cdots \mathrm{O} 2^{\mathrm{i}} / \mathrm{O} 2$ hydrogen bonds that form classical carboxylic-acid-based inversion dimers with $R_{2}^{2}(8)$ ring motifs. These contacts form chains propagating along [011] (yellow dashed lines in Fig. 2). In addition, intermolecular $\mathrm{N} 2-\mathrm{H} 2 A \cdots \mathrm{F} 1$ hydrogen bonds connect these chains, yielding sheets extending parallel to the $b c$ plane (red dashed line in Fig. 3). These sheets are further linked by weak intermolecular $\pi-\pi$ interactions between the pyridyl rings (N1/C1-C5) $\left[C g 1 \cdots C g 1^{\text {iv }}=3.4602(9) \AA\right.$; symmetry code: (iv) $-x,-y+2,-z]$, resulting in a three-dimensional network structure (black dashed lines in Fig. 4).

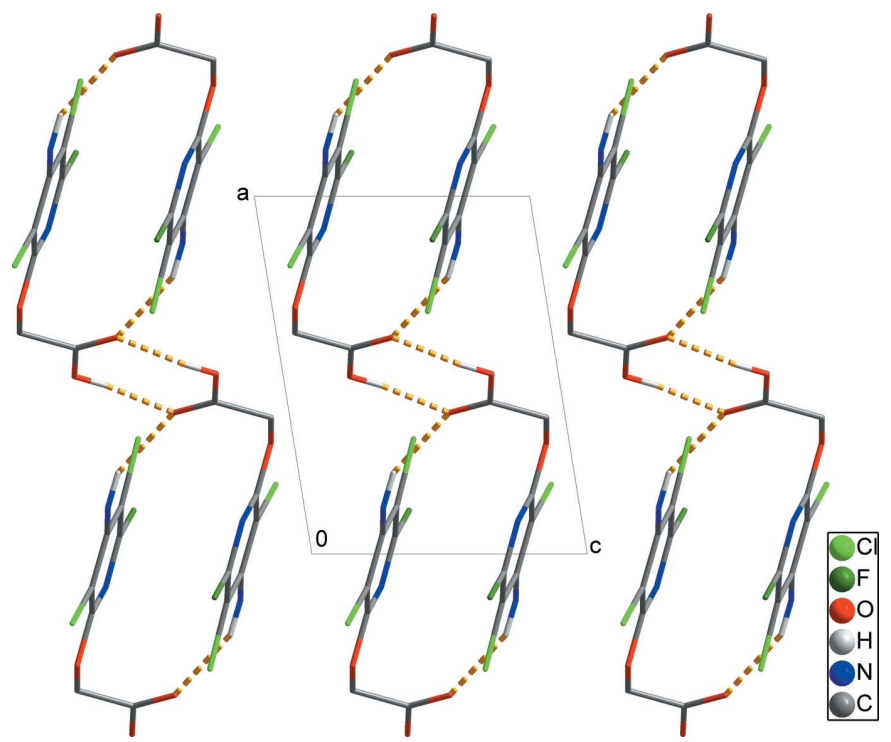

Figure 2

A view along the $b$ axis of the crystal packing of the title compound. The chains are formed through intermolecular $\mathrm{O}-\mathrm{H} \cdots \mathrm{O}$ and $\mathrm{N}-\mathrm{H} \cdots \mathrm{O}$ hydrogen bonds (yellow dashed lines). $\mathrm{H}$ atoms not involved in these interactions have been omitted for clarity.
Table 1

Hydrogen-bond geometry $\left(\AA{ }^{\circ}{ }^{\circ}\right)$.

\begin{tabular}{lllll}
\hline$D-\mathrm{H} \cdots A$ & $D-\mathrm{H}$ & $\mathrm{H} \cdots A$ & $D \cdots A$ & $D-\mathrm{H} \cdots A$ \\
\hline $\mathrm{O} 3-\mathrm{H} 3 \cdots \mathrm{O} 2^{\mathrm{i}}$ & 0.84 & 1.84 & $2.6801(15)$ & 174 \\
$\mathrm{~N} 2-\mathrm{H} 2 A \cdots \mathrm{F} 1^{\mathrm{ii}}$ & 0.88 & 2.39 & $2.9950(15)$ & 126 \\
$\mathrm{~N} 2-\mathrm{H} 2 B \cdots \mathrm{O} 2{ }^{\mathrm{iii}}$ & 0.88 & 2.25 & $3.0201(16)$ & 146 \\
\hline
\end{tabular}

Symmetry codes: (i) $-x+1,-y+1,-z+1$; (ii) $x, y+1, z$; (iii) $-x+1,-y+2,-z$.

\section{Database survey}

We have reported the crystal structure of several pesticides including compounds with pyridine rings (Cho et al., 2015; Kang et al., 2015; Kwon et al., 2016; Park et al., 2016). In addition, a database search (CSD; Groom et al., 2006) yielded

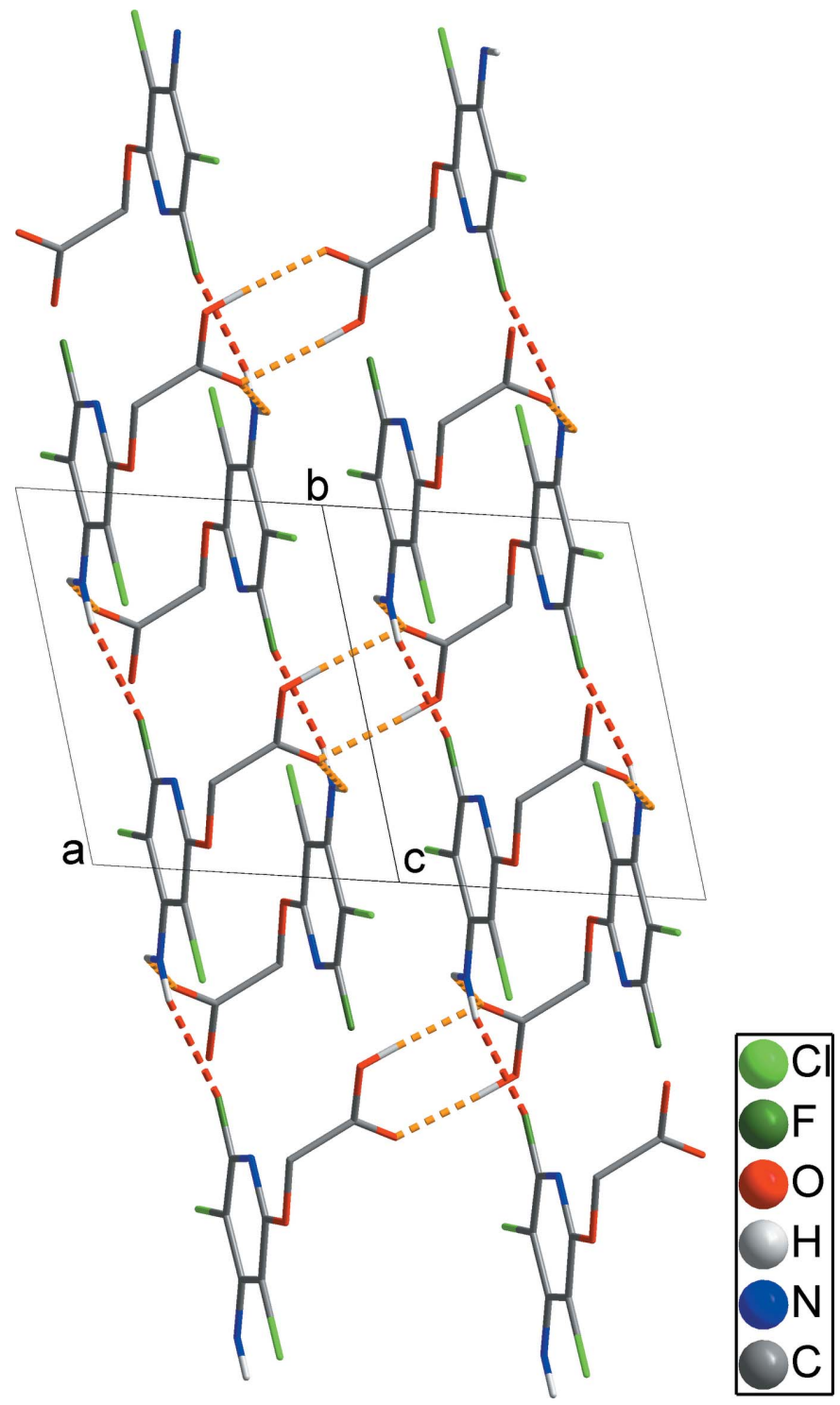

Figure 3

The two-dimensional network formed through intermolecular $\mathrm{N}-\mathrm{H} \cdots \mathrm{F}$ hydrogen bonds (red dashed lines). Intermolecular $\mathrm{O} / \mathrm{N}-\mathrm{H} \cdots \mathrm{O}$ hydrogen bonds within a chain are shown as yellow dashed lines. $\mathrm{H}$ atoms not involved in these interactions have been omitted for clarity. 


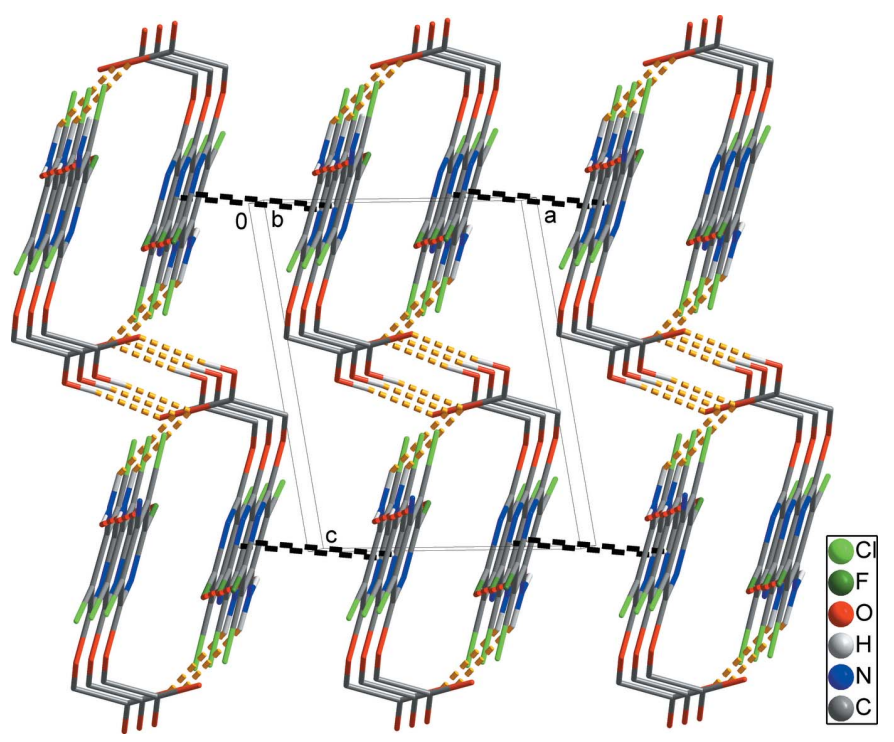

Figure 4

A packing diagram showing the three-dimensional architecture formed by weak $\pi-\pi$ interactions (black dashed lines). Intermolecular $\mathrm{O}-$ $\mathrm{H} \cdots \mathrm{O}, \mathrm{N}-\mathrm{H} \cdots \mathrm{O}$ and $\mathrm{N}-\mathrm{H} \cdots \mathrm{F}$ hydrogen bonds within a sheet are shown as yellow and red dashed lines. $\mathrm{H}$ atoms not involved in intermolecular interactions have been omitted for clarity.

two other comparable structures, 2-[(3,5,6-trichloropyridin-2yl)oxy]acetic acid (Cho et al., 2014) and 2,4,5-trichlorophenoxyacetic acid (Smith et al., 1976).

\section{Synthesis and crystallization}

The title compound was purchased from Dr. Ehrenstorfer $\mathrm{GmbH}$. Colorless single crystals suitable for X-ray diffraction were obtained from a $\mathrm{CH}_{3} \mathrm{CN}$ solution by slow evaporation at room temperature.

\section{Refinement}

Crystal data, data collection and structure refinement details are summarized in Table 2. All $\mathrm{H}$ atoms were positioned geometrically and refined using a riding model with $d(\mathrm{O}-\mathrm{H})$ $=0.84 \AA, U_{\text {iso }}=1.5 U_{\text {eq }}(\mathrm{C})$ for the $\mathrm{O}-\mathrm{H}$ group, $d(\mathrm{~N}-\mathrm{H})=$ $0.88 \AA, U_{\text {iso }}=1.2 U_{\text {eq }}(\mathrm{C})$ for the amine group, and $d(\mathrm{C}-\mathrm{H})=$ $0.99 \AA, U_{\text {iso }}=1.2 U_{\text {eq }}(\mathrm{C})$ for the $\mathrm{CH}_{2}$ group.

\section{Acknowledgements}

This research was supported by the Basic Science Research Program through the National Research Foundation of Korea (NRF) funded by the Ministry of Education, Science and Technology (Nos. 2014R1A1A4A01009105 and 2016R1D1A1B03934376).
Table 2

Experimental details.

\begin{tabular}{|c|c|}
\hline \multicolumn{2}{|l|}{ Crystal data } \\
\hline Chemical formula & $\mathrm{C}_{7} \mathrm{H}_{5} \mathrm{Cl}_{2} \mathrm{FN}_{2} \mathrm{O}_{3}$ \\
\hline$M_{\mathrm{r}}$ & 255.03 \\
\hline Crystal system, space group & Triclinic, $P \overline{1}$ \\
\hline Temperature (K) & 173 \\
\hline$a, b, c(\AA)$ & $7.1116(9), 7.6131(9), 8.9414(11)$ \\
\hline$\alpha, \beta, \gamma\left({ }^{\circ}\right)$ & $86.927(6), 80.354(6), 72.587(5)$ \\
\hline$V\left(\AA^{3}\right)$ & $455.38(10)$ \\
\hline$Z$ & 2 \\
\hline Radiation type & Мo $K \alpha$ \\
\hline$\mu\left(\mathrm{mm}^{-1}\right)$ & 0.72 \\
\hline Crystal size $(\mathrm{mm})$ & $0.23 \times 0.22 \times 0.04$ \\
\hline \multicolumn{2}{|l|}{ Data collection } \\
\hline Diffractometer & Bruker APEXII CCD \\
\hline Absorption correction & $\begin{array}{l}\text { Multi-scan (SADABS; Bruker, } \\
\text { 2014) }\end{array}$ \\
\hline$T_{\min }, T_{\max }$ & $0.690,0.746$ \\
\hline $\begin{array}{l}\text { No. of measured, independent and } \\
\text { observed }[I>2 \sigma(I)] \text { reflections }\end{array}$ & $8052,2092,1972$ \\
\hline$R_{\mathrm{int}}$ & 0.023 \\
\hline$(\sin \theta / \lambda)_{\max }\left(\AA^{-1}\right)$ & 0.650 \\
\hline \multicolumn{2}{|l|}{ Refinement } \\
\hline$R\left[F^{2}>2 \sigma\left(F^{2}\right)\right], w R\left(F^{2}\right), S$ & $0.028,0.076,1.12$ \\
\hline No. of reflections & 2092 \\
\hline No. of parameters & 137 \\
\hline $\mathrm{H}$-atom treatment & $\mathrm{H}$-atom parameters constrained \\
\hline$\Delta \rho_{\max }, \Delta \rho_{\min }\left(\mathrm{e} \AA^{-3}\right)$ & $0.29,-0.38$ \\
\hline
\end{tabular}

Computer programs: APEX2 and SAINT (Bruker, 2014), SHELXS97 and SHELXTL (Sheldrick, 2008), SHELXL2014 (Sheldrick, 2015) and DIAMOND (Brandenburg, 2010).

\section{References}

Brandenburg, K. (2010). DIAMOND. Crystal Impact GbR, Bonn, Germany.

Bruker (2014). APEX2, SAINT and SADABS. Bruker AXS Inc., Madison, Wisconsin, USA.

Cho, S., Kim, J., Jeon, Y. \& Kim, T. H. (2014). Acta Cryst. E70, o940.

Cho, S., Kim, J., Lee, S. \& Kim, T. H. (2015). Acta Cryst. E71, o55.

Groom, C. R., Bruno, I. J., Lightfoot, M. P. \& Ward, S. C. (2016). Acta Cryst. B72, 171-179.

Kang, G., Kim, J., Park, H. \& Kim, T. H. (2015). Acta Cryst. E71, 0588.

Kwon, E., Kim, J., Park, H. \& Kim, T. H. (2016). Acta Cryst. E72, 1468-1470.

Moreno-Castilla, C., López-Ramón, M. V., Pastrana-Martínez, L. M., Álvarez-Merino, M. A. \& Fontecha-Cámara, M. A. (2012). Adsorption, 18, 173-179.

Park, H., Kwon, E., Yoon, I. \& Kim, J. (2016). Acta Cryst. E72, 16101613.

Reed, T. V. \& McCullough, P. E. (2012). Hort. Sci. 47, 1548-1549.

Sheldrick, G. M. (2008). Acta Cryst. A64, 112-122.

Sheldrick, G. M. (2015). Acta Cryst. C71, 3-8.

Smith, G., Kennard, C. H. L. \& White, A. H. (1976). Aust. J. Chem. 29, 2727-2730.

Wang, L., Xu, J., Zhao, P. \& Pan, C. (2011). Bull. Environ. Contam. Toxicol. 86, 449-453. 


\section{supporting information}

Acta Cryst. (2016). E72, 1836-1838 [https://doi.org/10.1107/S2056989016018533]

\section{Crystal structure of fluroxypyr}

\section{Hyunjin Park, Myong Yong Choi, Eunjin Kwon and Tae Ho Kim}

\section{Computing details}

Data collection: APEX2 (Bruker, 2014); cell refinement: SAINT (Bruker, 2014); data reduction: SAINT (Bruker, 2014); program(s) used to solve structure: SHELXS97 (Sheldrick, 2008); program(s) used to refine structure: SHELXL2014 (Sheldrick, 2015); molecular graphics: DIAMOND (Brandenburg, 2010); software used to prepare material for publication: SHELXTL (Sheldrick, 2008).

2-[(4-Amino-3,5-dichloro-6-fluoropyridin-2-yl)oxy]acetic acid

Crystal data

$\mathrm{C}_{7} \mathrm{H}_{5} \mathrm{Cl}_{2} \mathrm{FN}_{2} \mathrm{O}_{3}$

$M_{r}=255.03$

Triclinic, $P \overline{1}$

$a=7.1116(9) \AA$

$b=7.6131(9) \AA$

$c=8.9414(11) \AA$

$\alpha=86.927(6)^{\circ}$

$\beta=80.354(6)^{\circ}$

$\gamma=72.587(5)^{\circ}$

$V=455.38(10) \AA^{3}$

Data collection

Bruker APEXII CCD

diffractometer

$\varphi$ and $\omega$ scans

Absorption correction: multi-scan

(SADABS; Bruker, 2014)

$T_{\min }=0.690, T_{\max }=0.746$

8052 measured reflections

Refinement

Refinement on $F^{2}$

Least-squares matrix: full

$R\left[F^{2}>2 \sigma\left(F^{2}\right)\right]=0.028$

$w R\left(F^{2}\right)=0.076$

$S=1.12$

2092 reflections

137 parameters

0 restraints
$Z=2$

$F(000)=256$

$D_{\mathrm{x}}=1.860 \mathrm{Mg} \mathrm{m}^{-3}$

Mo $K \alpha$ radiation, $\lambda=0.71073 \AA$

Cell parameters from 6070 reflections

$\theta=2.8-27.5^{\circ}$

$\mu=0.72 \mathrm{~mm}^{-1}$

$T=173 \mathrm{~K}$

Plate, colourless

$0.23 \times 0.22 \times 0.04 \mathrm{~mm}$

2092 independent reflections

1972 reflections with $I>2 \sigma(I)$

$R_{\text {int }}=0.023$

$\theta_{\text {max }}=27.5^{\circ}, \theta_{\min }=2.3^{\circ}$

$h=-9 \rightarrow 8$

$k=-9 \rightarrow 9$

$l=-10 \rightarrow 11$

Hydrogen site location: inferred from neighbouring sites

$\mathrm{H}$-atom parameters constrained

$w=1 /\left[\sigma^{2}\left(F_{\mathrm{o}}^{2}\right)+(0.0371 P)^{2}+0.2062 P\right]$

where $P=\left(F_{\mathrm{o}}{ }^{2}+2 F_{\mathrm{c}}{ }^{2}\right) / 3$

$(\Delta / \sigma)_{\max }<0.001$

$\Delta \rho_{\max }=0.29 \mathrm{e} \AA^{-3}$

$\Delta \rho_{\min }=-0.38$ e $\AA^{-3}$ 


\section{Special details}

Geometry. All esds (except the esd in the dihedral angle between two 1.s. planes) are estimated using the full covariance matrix. The cell esds are taken into account individually in the estimation of esds in distances, angles and torsion angles; correlations between esds in cell parameters are only used when they are defined by crystal symmetry. An approximate (isotropic) treatment of cell esds is used for estimating esds involving l.s. planes.

Fractional atomic coordinates and isotropic or equivalent isotropic displacement parameters $\left(\AA^{2}\right)$

\begin{tabular}{lllll}
\hline & $x$ & $y$ & $z$ & $U_{\text {iso }} / U_{\text {eq }}$ \\
\hline C11 & $0.43701(6)$ & $0.90760(5)$ & $-0.32138(4)$ & $0.02990(12)$ \\
C12 & $0.09018(6)$ & $1.29195(5)$ & $0.19394(4)$ & $0.02692(11)$ \\
F1 & $0.37852(14)$ & $0.60390(12)$ & $-0.12163(11)$ & $0.0298(2)$ \\
O1 & $0.08333(16)$ & $0.93076(14)$ & $0.31061(11)$ & $0.0234(2)$ \\
O2 & $0.41952(15)$ & $0.68493(14)$ & $0.39455(11)$ & $0.0225(2)$ \\
O3 & $0.24193(16)$ & $0.50096(15)$ & $0.50373(13)$ & $0.0278(2)$ \\
H3 & 0.3515 & 0.4408 & 0.5295 & $0.042^{*}$ \\
N1 & $0.23296(18)$ & $0.76320(16)$ & $0.09169(14)$ & $0.0202(2)$ \\
N2 & $0.2865(2)$ & $1.24580(17)$ & $-0.12976(14)$ & $0.0244(3)$ \\
H2A & 0.2414 & 1.3515 & -0.0813 & $0.029^{*}$ \\
H2B & 0.3429 & 1.2417 & -0.2255 & $0.029^{*}$ \\
C1 & $0.3133(2)$ & $0.76709(19)$ & $-0.05118(17)$ & $0.0202(3)$ \\
C2 & $0.3356(2)$ & $0.91926(19)$ & $-0.13244(15)$ & $0.0192(3)$ \\
C3 & $0.26931(19)$ & $1.08983(18)$ & $-0.05718(15)$ & $0.0177(3)$ \\
C4 & $0.1811(2)$ & $1.08826(18)$ & $0.09480(15)$ & $0.0176(3)$ \\
C5 & $0.16793(19)$ & $0.92352(19)$ & $0.16331(15)$ & $0.0178(3)$ \\
C6 & $0.0683(2)$ & $0.7624(2)$ & $0.38160(17)$ & $0.0242(3)$ \\
H6A & 0.0248 & 0.6917 & 0.3114 & $0.029^{*}$ \\
H6B & -0.0345 & 0.7900 & 0.4736 & $0.029^{*}$ \\
C7 & $0.2634(2)$ & $0.64680(19)$ & $0.42518(15)$ & $0.0197(3)$ \\
\end{tabular}

Atomic displacement parameters $\left(\AA^{2}\right)$

\begin{tabular}{lllllll}
\hline & $U^{11}$ & $U^{22}$ & $U^{33}$ & $U^{12}$ & $U^{13}$ & $U^{23}$ \\
\hline C11 & $0.0345(2)$ & $0.0351(2)$ & $0.01671(18)$ & $-0.00767(16)$ & $0.00124(14)$ & $-0.00224(14)$ \\
C12 & $0.0359(2)$ & $0.01784(17)$ & $0.02395(19)$ & $-0.00397(14)$ & $-0.00232(14)$ & $-0.00355(13)$ \\
F1 & $0.0372(5)$ & $0.0182(4)$ & $0.0318(5)$ & $-0.0048(4)$ & $-0.0035(4)$ & $-0.0076(4)$ \\
O1 & $0.0270(5)$ & $0.0214(5)$ & $0.0177(5)$ & $-0.0044(4)$ & $0.0008(4)$ & $0.0058(4)$ \\
O2 & $0.0245(5)$ & $0.0241(5)$ & $0.0208(5)$ & $-0.0101(4)$ & $-0.0048(4)$ & $0.0060(4)$ \\
O3 & $0.0243(5)$ & $0.0232(5)$ & $0.0355(6)$ & $-0.0081(4)$ & $-0.0051(5)$ & $0.0130(4)$ \\
N1 & $0.0209(6)$ & $0.0169(5)$ & $0.0232(6)$ & $-0.0058(4)$ & $-0.0055(5)$ & $0.0035(4)$ \\
N2 & $0.0318(7)$ & $0.0200(6)$ & $0.0216(6)$ & $-0.0103(5)$ & $-0.0013(5)$ & $0.0055(5)$ \\
C1 & $0.0195(6)$ & $0.0167(6)$ & $0.0242(7)$ & $-0.0035(5)$ & $-0.0058(5)$ & $-0.0025(5)$ \\
C2 & $0.0190(6)$ & $0.0226(7)$ & $0.0158(6)$ & $-0.0060(5)$ & $-0.0025(5)$ & $-0.0002(5)$ \\
C3 & $0.0170(6)$ & $0.0189(6)$ & $0.0183(6)$ & $-0.0065(5)$ & $-0.0053(5)$ & $0.0037(5)$ \\
C4 & $0.0186(6)$ & $0.0155(6)$ & $0.0181(6)$ & $-0.0038(5)$ & $-0.0035(5)$ & $0.0002(5)$ \\
C5 & $0.0150(6)$ & $0.0200(6)$ & $0.0173(6)$ & $-0.0036(5)$ & $-0.0037(5)$ & $0.0033(5)$ \\
C6 & $0.0236(7)$ & $0.0258(7)$ & $0.0218(7)$ & $-0.0081(6)$ & $-0.0014(5)$ & $0.0090(6)$ \\
C7 & $0.0252(7)$ & $0.0202(6)$ & $0.0132(6)$ & $-0.0073(5)$ & $-0.0005(5)$ & $0.0007(5)$ \\
& & & & & &
\end{tabular}


Geometric parameters $(\AA, \stackrel{\circ}{)})$

\begin{tabular}{|c|c|c|c|}
\hline $\mathrm{C} 11-\mathrm{C} 2$ & $1.7181(14)$ & $\mathrm{N} 2-\mathrm{C} 3$ & $1.3506(17)$ \\
\hline $\mathrm{Cl} 2-\mathrm{C} 4$ & $1.7216(14)$ & $\mathrm{N} 2-\mathrm{H} 2 \mathrm{~A}$ & 0.8800 \\
\hline $\mathrm{F} 1-\mathrm{C} 1$ & $1.3403(16)$ & $\mathrm{N} 2-\mathrm{H} 2 \mathrm{~B}$ & 0.8800 \\
\hline $\mathrm{O} 1-\mathrm{C} 5$ & $1.3499(17)$ & $\mathrm{C} 1-\mathrm{C} 2$ & $1.370(2)$ \\
\hline $\mathrm{O} 1-\mathrm{C} 6$ & $1.4243(17)$ & $\mathrm{C} 2-\mathrm{C} 3$ & $1.4080(19)$ \\
\hline $\mathrm{O} 2-\mathrm{C} 7$ & $1.2143(17)$ & $\mathrm{C} 3-\mathrm{C} 4$ & $1.3990(19)$ \\
\hline $\mathrm{O} 3-\mathrm{C} 7$ & $1.3158(17)$ & $\mathrm{C} 4-\mathrm{C} 5$ & $1.3877(19)$ \\
\hline $\mathrm{O} 3-\mathrm{H} 3$ & 0.8400 & $\mathrm{C} 6-\mathrm{C} 7$ & $1.505(2)$ \\
\hline $\mathrm{N} 1-\mathrm{C} 1$ & $1.3117(19)$ & C6-H6A & 0.9900 \\
\hline $\mathrm{N} 1-\mathrm{C} 5$ & $1.3268(18)$ & $\mathrm{C} 6-\mathrm{H} 6 \mathrm{~B}$ & 0.9900 \\
\hline $\mathrm{C} 5-\mathrm{O} 1-\mathrm{C} 6$ & $117.32(11)$ & $\mathrm{C} 5-\mathrm{C} 4-\mathrm{C} 3$ & $119.78(12)$ \\
\hline $\mathrm{C} 7-\mathrm{O} 3-\mathrm{H} 3$ & 109.5 & $\mathrm{C} 5-\mathrm{C} 4-\mathrm{Cl} 2$ & $121.01(11)$ \\
\hline $\mathrm{C} 1-\mathrm{N} 1-\mathrm{C} 5$ & $116.03(12)$ & $\mathrm{C} 3-\mathrm{C} 4-\mathrm{Cl} 2$ & $119.20(10)$ \\
\hline $\mathrm{C} 3-\mathrm{N} 2-\mathrm{H} 2 \mathrm{~A}$ & 120.0 & $\mathrm{~N} 1-\mathrm{C} 5-\mathrm{O} 1$ & $119.54(12)$ \\
\hline $\mathrm{C} 3-\mathrm{N} 2-\mathrm{H} 2 \mathrm{~B}$ & 120.0 & $\mathrm{~N} 1-\mathrm{C} 5-\mathrm{C} 4$ & $123.51(13)$ \\
\hline $\mathrm{H} 2 \mathrm{~A}-\mathrm{N} 2-\mathrm{H} 2 \mathrm{~B}$ & 120.0 & $\mathrm{O} 1-\mathrm{C} 5-\mathrm{C} 4$ & $116.96(12)$ \\
\hline $\mathrm{N} 1-\mathrm{C} 1-\mathrm{F} 1$ & $115.22(12)$ & $\mathrm{O} 1-\mathrm{C} 6-\mathrm{C} 7$ & $112.12(12)$ \\
\hline $\mathrm{N} 1-\mathrm{C} 1-\mathrm{C} 2$ & $126.49(13)$ & $\mathrm{O} 1-\mathrm{C} 6-\mathrm{H} 6 \mathrm{~A}$ & 109.2 \\
\hline $\mathrm{F} 1-\mathrm{C} 1-\mathrm{C} 2$ & $118.29(13)$ & $\mathrm{C} 7-\mathrm{C} 6-\mathrm{H} 6 \mathrm{~A}$ & 109.2 \\
\hline $\mathrm{C} 1-\mathrm{C} 2-\mathrm{C} 3$ & $118.00(13)$ & $\mathrm{O} 1-\mathrm{C} 6-\mathrm{H} 6 \mathrm{~B}$ & 109.2 \\
\hline $\mathrm{C} 1-\mathrm{C} 2-\mathrm{Cl} 1$ & $122.11(11)$ & $\mathrm{C} 7-\mathrm{C} 6-\mathrm{H} 6 \mathrm{~B}$ & 109.2 \\
\hline $\mathrm{C} 3-\mathrm{C} 2-\mathrm{C} 11$ & $119.88(10)$ & $\mathrm{H} 6 \mathrm{~A}-\mathrm{C} 6-\mathrm{H} 6 \mathrm{~B}$ & 107.9 \\
\hline $\mathrm{N} 2-\mathrm{C} 3-\mathrm{C} 4$ & $122.43(12)$ & $\mathrm{O} 2-\mathrm{C} 7-\mathrm{O} 3$ & $124.35(13)$ \\
\hline $\mathrm{N} 2-\mathrm{C} 3-\mathrm{C} 2$ & $121.39(12)$ & $\mathrm{O} 2-\mathrm{C} 7-\mathrm{C} 6$ & $124.46(13)$ \\
\hline $\mathrm{C} 4-\mathrm{C} 3-\mathrm{C} 2$ & $116.17(12)$ & $\mathrm{O} 3-\mathrm{C} 7-\mathrm{C} 6$ & $111.18(12)$ \\
\hline $\mathrm{C} 5-\mathrm{N} 1-\mathrm{C} 1-\mathrm{F} 1$ & $179.85(11)$ & $\mathrm{C} 2-\mathrm{C} 3-\mathrm{C} 4-\mathrm{Cl} 2$ & $-178.35(10)$ \\
\hline $\mathrm{C} 5-\mathrm{N} 1-\mathrm{C} 1-\mathrm{C} 2$ & $-0.2(2)$ & $\mathrm{C} 1-\mathrm{N} 1-\mathrm{C} 5-\mathrm{O} 1$ & $179.97(12)$ \\
\hline $\mathrm{N} 1-\mathrm{C} 1-\mathrm{C} 2-\mathrm{C} 3$ & $0.9(2)$ & $\mathrm{C} 1-\mathrm{N} 1-\mathrm{C} 5-\mathrm{C} 4$ & $0.1(2)$ \\
\hline $\mathrm{F} 1-\mathrm{C} 1-\mathrm{C} 2-\mathrm{C} 3$ & $-179.10(12)$ & $\mathrm{C} 6-\mathrm{O} 1-\mathrm{C} 5-\mathrm{N} 1$ & $-0.13(18)$ \\
\hline $\mathrm{N} 1-\mathrm{C} 1-\mathrm{C} 2-\mathrm{C} 11$ & $-178.05(11)$ & $\mathrm{C} 6-\mathrm{O} 1-\mathrm{C} 5-\mathrm{C} 4$ & $179.71(12)$ \\
\hline $\mathrm{F} 1-\mathrm{C} 1-\mathrm{C} 2-\mathrm{Cl} 1$ & 1.94 (19) & $\mathrm{C} 3-\mathrm{C} 4-\mathrm{C} 5-\mathrm{N} 1$ & $-0.9(2)$ \\
\hline $\mathrm{C} 1-\mathrm{C} 2-\mathrm{C} 3-\mathrm{N} 2$ & $179.92(13)$ & $\mathrm{Cl} 2-\mathrm{C} 4-\mathrm{C} 5-\mathrm{N} 1$ & $179.01(10)$ \\
\hline $\mathrm{C} 11-\mathrm{C} 2-\mathrm{C} 3-\mathrm{N} 2$ & $-1.09(18)$ & $\mathrm{C} 3-\mathrm{C} 4-\mathrm{C} 5-\mathrm{O} 1$ & $179.27(11)$ \\
\hline $\mathrm{C} 1-\mathrm{C} 2-\mathrm{C} 3-\mathrm{C} 4$ & $-1.55(19)$ & $\mathrm{Cl} 2-\mathrm{C} 4-\mathrm{C} 5-\mathrm{O} 1$ & $-0.82(17)$ \\
\hline $\mathrm{C} 11-\mathrm{C} 2-\mathrm{C} 3-\mathrm{C} 4$ & $177.43(10)$ & $\mathrm{C} 5-\mathrm{O} 1-\mathrm{C} 6-\mathrm{C} 7$ & $78.48(15)$ \\
\hline $\mathrm{N} 2-\mathrm{C} 3-\mathrm{C} 4-\mathrm{C} 5$ & $-179.93(12)$ & $\mathrm{O} 1-\mathrm{C} 6-\mathrm{C} 7-\mathrm{O} 2$ & $-4.9(2)$ \\
\hline $\mathrm{C} 2-\mathrm{C} 3-\mathrm{C} 4-\mathrm{C} 5$ & $1.56(19)$ & $\mathrm{O} 1-\mathrm{C} 6-\mathrm{C} 7-\mathrm{O} 3$ & $173.75(12)$ \\
\hline $\mathrm{N} 2-\mathrm{C} 3-\mathrm{C} 4-\mathrm{Cl} 2$ & $0.16(18)$ & & \\
\hline
\end{tabular}

Hydrogen-bond geometry $\left(\AA,{ }^{\circ}\right)$

\begin{tabular}{lllll}
\hline$D-\mathrm{H} \cdots A$ & $D-\mathrm{H}$ & $\mathrm{H} \cdots A$ & $D \cdots A$ & $D-\mathrm{H} \cdots A$ \\
\hline $\mathrm{O} 3-\mathrm{H} 3 \cdots \mathrm{O} 2^{\mathrm{i}}$ & 0.84 & 1.84 & $2.6801(15)$ & 174
\end{tabular}


supporting information

$\begin{array}{lllll}\mathrm{N} 2-\mathrm{H} 2 A \cdots \mathrm{F} 1^{\mathrm{ii}} & 0.88 & 2.39 & 2.9950(15) & 126 \\ \mathrm{~N} 2-\mathrm{H} 2 B \cdots \mathrm{O} 2^{\mathrm{iii}} & 0.88 & 2.25 & 3.0201(16) & 146\end{array}$

Symmetry codes: (i) $-x+1,-y+1,-z+1$; (ii) $x, y+1, z$; (iii) $-x+1,-y+2,-z$. 